\title{
A NEW SUBCLASS OF HARMONIC UNIVALENT FUNCTIONS DEFINED BY FRACTIONAL CALCULUS OPERATOR WITH MISSING COEFFICIENTS
}

\author{
B. Singh, S. Porwal
}

ABstRACT. In this paper, authors introduce and study a new subclass of harmonic univalent functions. Coefficient conditions, extreme points, distortion bounds, convolution and convex combination for this class are obtained. Relevant connections of the results presented here with various well-known results are briefly indicated.

2010 Mathematics Subject Classification: 30C45.

Keywords: Harmonic, Univalent functions, Fractional Calculus.

\section{INTRODUCTION}

A continuous complex-valued function $f=u+i v$ defined in a simply-connected complex domain $D$ is said to be harmonic in $D$ if both $u$ and $v$ are real harmonic in $D$. In any simply connected domain we can write $f=h+\bar{g}$ where $h$ and $g$ are analytic in $D$. We call $h$ the analytic part and $g$ the co-analytic part of $f$. A necessary and sufficient condition for $f$ to be locally univalent and sense-preserving in $D$ is that $\left|h^{\prime}(z)\right|>\left|g^{\prime}(z)\right|, z \in D$. See Clunie and Sheil-Small [2].

Denote by $S_{H}$ the class of functions $f=h+\bar{g}$ that are harmonic univalent and sense-preserving in the open unit disk $U=\{z:|z|<1\}$ for which $f(0)=f_{z}(0)-1=$ 0 . Then for $f=h+\bar{g} \in S_{H}$ we may express the analytic functions $h$ and $g$ as

$$
h(z)=z+\sum_{k=2}^{\infty} a_{k} z^{k}, \quad g(z)=\sum_{k=1}^{\infty} b_{k} z^{k}, \quad\left|b_{1}\right|<1 .
$$

Further, we denote by $S_{H}^{j}$ the subclass of $S_{H}$ consisting of the functions $f=h+\bar{g}$ where $h$ and $g$ are of the form

$$
h(z)=z+\sum_{k=j+1}^{\infty} a_{k} z^{k}, \quad g(z)=\sum_{k=1}^{\infty} b_{k} z^{k}, \quad j \in N, \quad\left|b_{1}\right|<1 .
$$


B. Singh, S. Porwal - A new subclass of harmonic univalent functions ...

Recently, Dixit and Porwal [3](see also [6], [7]) introduce the operator $\Omega^{n}$ for function $f(z)$ of the form (1) as

$$
\Omega^{n} f(z)=\Omega^{n} h(z)+(-1)^{n} \overline{\Omega^{n} g(z)}
$$

where $\Omega^{n} h(z)=z+\sum_{k=2}^{\infty}[\phi(k, \lambda)]^{n} a_{k} z^{k}, \Omega^{n} g(z)=\sum_{k=1}^{\infty}[\phi(k, \lambda)]^{n} b_{k} z^{k}$. and $\phi(k, \lambda)=$ $\frac{\Gamma(k+1) \Gamma(1-\lambda)}{\Gamma(k-\lambda)}$.

Now for $0 \leq \alpha<1,0 \leq \lambda<1, m \in N, n \in N_{0}, m>n$ and $z \in U$, suppose that $S_{H}^{\lambda}(m, n, j ; \alpha)$ denote the family of harmonic functions $f$ of the form (2) such that

$$
\Re\left\{\frac{\Omega^{m} f(z)}{\Omega^{n} f(z)}\right\}>\alpha,
$$

where $\Omega^{m} f$ is defined by $(3)$.

Further, let the subclass $\bar{S}_{H}^{\lambda}(m, n, j ; \alpha)$ consist of harmonic functions $f_{m}=h+\overline{g_{m}}$ in $S_{H}^{\lambda}(m, n, j ; \alpha)$ so that $h$ and $g_{m}$ are the form

$$
h(z)=z-\sum_{k=j+1}^{\infty}\left|a_{k}\right| z^{k}, \quad g_{m}(z)=(-1)^{m-1} \sum_{k=1}^{\infty}\left|b_{k}\right| z^{k} .
$$

Assigning specific values to $m, n, j, \alpha$ and $\lambda$ in the subclass $S_{H}^{\lambda}(m, n, j ; \alpha)$ we obtain the following known subclasses studied earlier by various researchers.

1. If we put $j=1, \lambda=0$ then it reduces to the class $S_{H}(m, n ; \alpha)$ studied by Yalcin [10].

2. If we put $j=1, m=1, n=0, \lambda=0$ and $m=2, n=1, \lambda=0$ then it reduces to the class $H S(\alpha)$ and $H K(\alpha)$ studied by Jahangiri [4].

3. If we put $j=1, m=1, n=0, \alpha=0, \lambda=0$ and $m=2, n=1, \alpha=0, \lambda=0$ with $b_{1}=0$ then it reduces to the class $H S^{0}(0)$ and $H K^{0}(0)$ studied by Avci and Zlotkiewicz [1] and Silverman [8].

4. If we put $j=1, m=1, n=0, \alpha=0, \lambda=0$ and $m=2, n=1, \alpha=0, \lambda=0$ then it reduces to the class $H S(0)$ and $H K(0)$ studied by Silverman and Silvia [9], which is an improvement of ([1], [8]).

5. If we put $j=1, m=n+1, \lambda=0$ then it reduces to the class $H(n, \alpha)$ studied by Jahangiri et al. [5].

In the present paper, results involving coefficient estimates, extreme points, distortion bounds, convolution condition and convex combinations for the above classes $S_{H}^{\lambda}(m, n, j ; \alpha)$ and $\bar{S}_{H}^{\lambda}(m, n, j ; \alpha)$ of harmonic univalent functions have been investigated. 
B. Singh, S. Porwal - A new subclass of harmonic univalent functions ...

\section{MAin Results}

We begin with a sufficient coefficient condition for function in $S_{H}^{\lambda}(m, n, j ; \alpha)$.

Theorem 1. Let $f=h+\bar{g}$ be such that $h$ and $g$ are given by (2). Furthermore, let

$$
\sum_{k=j+1}^{\infty} \frac{[\phi(k, \lambda)]^{m}-\alpha[\phi(k, \lambda)]^{n}}{1-\alpha}\left|a_{k}\right|+\sum_{k=1}^{\infty} \frac{[\phi(k, \lambda)]^{m}-(-1)^{m-n} \alpha[\phi(k, \lambda)]^{n}}{1-\alpha}\left|b_{k}\right| \leq 1
$$

where $a_{1}=1, m, j \in N, n \in N_{0}, m>n, 0 \leq \alpha<1,0 \leq \lambda<1$ and $\phi(k, \lambda)=\frac{\Gamma(k+1) \Gamma(1-\lambda)}{\Gamma(k-\lambda)}$, then $f$ is sense-preserving, harmonic univalent in $U$ and $f \in S_{H}^{\lambda}(m, n, j ; \alpha)$.

Proof. First we note that $f$ is sense-preserving in $U$. This is because

$$
\begin{aligned}
\left|h^{\prime}(z)\right| & \geq 1-\sum_{k=j+1}^{\infty} k\left|a_{k}\right| r^{k-1} \\
& >1-\sum_{k=j+1}^{\infty} k\left|a_{k}\right| \\
& \geq 1-\sum_{k=j+1}^{\infty} \frac{[\phi(k, \lambda)]^{m}-\alpha[\phi(k, \lambda)]^{n}}{1-\alpha}\left|a_{k}\right| \\
& \geq \sum_{k=1}^{\infty} \frac{[\phi(k, \lambda)]^{m}-(-1)^{m-n} \alpha[\phi(k, \lambda)]^{n}}{1-\alpha}\left|b_{k}\right| \\
& \geq \sum_{k=1}^{\infty} k\left|b_{k}\right| \\
& >\sum_{k=1}^{\infty} k\left|b_{k}\right| r^{k-1} \\
& \geq\left|g^{\prime}(z)\right| .
\end{aligned}
$$


B. Singh, S. Porwal - A new subclass of harmonic univalent functions ...

To show that $f$ is univalent $U$, suppose $z_{1}, z_{2} \in U$ such that $z_{1} \neq z_{2}$, then

$$
\begin{aligned}
\left|\frac{f\left(z_{1}\right)-f\left(z_{2}\right)}{h\left(z_{1}\right)-h\left(z_{2}\right)}\right| & \geq 1-\left|\frac{g\left(z_{1}\right)-g\left(z_{2}\right)}{h\left(z_{1}\right)-h\left(z_{2}\right)}\right| \\
& =1-\left|\frac{\sum_{k=1}^{\infty} b_{k}\left(z_{1}^{k}-z_{2}^{k}\right)}{z_{1}-z_{2}+\sum_{k=j+1}^{\infty} a_{k}\left(z_{1}^{k}-z_{2}^{k}\right)}\right| \\
& >1-\frac{\sum_{k=1}^{\infty} k\left|b_{k}\right|}{1-\sum_{k=j+1}^{\infty} k\left|a_{k}\right|} \\
& \geq 1-\frac{\sum_{k=1}^{\infty} \frac{[\phi(k, \lambda)]^{m}-(-1)^{m-n} \alpha[\phi(k, \lambda)]^{n}}{1-\alpha}\left|b_{k}\right|}{1-\sum_{k=j+1}^{\infty} \frac{[\phi(k, \lambda)]^{m}-\alpha[\phi(k, \lambda)]^{n}}{1-\alpha}\left|a_{k}\right|} \\
& \geq 0 .
\end{aligned}
$$

Now, we show that $f \in S_{H}^{\lambda}(m, n, j ; \alpha)$. Using the fact that $R e \omega \geq \alpha$, if and only if, $|1-\alpha+\omega| \geq|1+\alpha-\omega|$, it suffices to show that

$$
|A(z)+(1-\alpha) B(z)|-|A(z)-(1+\alpha) B(z)| \geq 0,
$$

where $A(z)=\Omega^{m} f(z)$ and $B(z)=\Omega^{n} f(z)$. 
B. Singh, S. Porwal - A new subclass of harmonic univalent functions ...

Substituting for $A(z)$ and $B(z)$ in L.H.S. of (7) and making use of (6), we obtain

$$
\begin{aligned}
& |A(z)+(1-\alpha) B(z)|-|A(z)-(1+\alpha) B(z)| \\
& =\left|\Omega^{m} f(z)+(1-\alpha) \Omega^{n} f(z)\right|-\left|\Omega^{m} f(z)-(1+\alpha) \Omega^{n} f(z)\right| \mid \\
& =\mid(2-\alpha) z+\sum_{k=j+1}^{\infty}\left([\phi(k, \lambda)]^{m}+(1-\alpha)[\phi(k, \lambda)]^{n}\right) a_{k} z^{k} \\
& +(-1)^{n} \sum_{k=1}^{\infty}\left((-1)^{m-n}[\phi(k, \lambda)]^{m}+(1-\alpha)[\phi(k, \lambda)]^{n}\right) \overline{b_{k} z^{k}} \\
& -\mid-\alpha z+\sum_{k=j+1}^{\infty}\left([\phi(k, \lambda)]^{m}-(1+\alpha)[\phi(k, \lambda)]^{n}\right) a_{k} z^{k} \\
& -(-1)^{n} \sum_{k=1}^{\infty}\left((-1)^{m-n}[\phi(k, \lambda)]^{m}-(1+\alpha)[\phi(k, \lambda)]^{n}\right) \overline{b_{k} z^{k}} \\
& \geq 2(1-\alpha)|z|-2 \sum_{k=j+1}^{\infty}\left([\phi(k, \lambda)]^{m}-\alpha[\phi(k, \lambda)]^{n}\right)\left|\alpha_{k}\right||z|^{k} \\
& -\sum_{k=1}^{\infty}\left|(-1)^{m-n}[\phi(k, \lambda)]^{m}+(1-\alpha)[\phi(k, \lambda)]^{n}\right|\left|b_{k}\right||z|^{k} \\
& -\sum_{k=1}^{\infty}\left|(-1)^{m-n}[\phi(k, \lambda)]^{m}-(1+\alpha)[\phi(k, \lambda)]^{n}\right|\left|b_{k}\right||z|^{k} \\
& \left(2(1-\alpha)|z|-2 \sum_{k=j+1}^{\infty}\left[(\phi(k, \lambda))^{m}-\alpha(\phi(k, \lambda))^{n}\right]\left|a_{k}\right||z|^{k}\right. \\
& \begin{array}{l}
-2 \sum_{k=1}^{\infty}\left[(\phi(k, \lambda))^{m}+\alpha(\phi(k, \lambda))^{n}\right]\left|b_{k}\right||z|^{k}, \text { if } m-n \text { is odd } \\
-\alpha)|z|-2 \sum_{k=j+1}^{\infty}\left[(\phi(k, \lambda))^{m}-\alpha(\phi(k, \lambda))^{n}\right]\left|a_{k}\right||z|^{k}
\end{array} \\
& -2 \sum_{k=1}^{\infty}\left[(\phi(k, \lambda))^{m}-\alpha(\phi(k, \lambda))^{n}\right]\left|b_{k}\right||z|^{k}, \text { if } m-n \text { is even }
\end{aligned}
$$


B. Singh, S. Porwal - A new subclass of harmonic univalent functions ...

$$
\begin{aligned}
& =2(1-\alpha)|z|\left\{1-\sum_{k=j+1}^{\infty} \frac{[\phi(k, \lambda)]^{m}-\alpha[\phi(k, \lambda)]^{n}}{1-\alpha}\left|a_{k}\right||z|^{k-1}\right. \\
& \left.-\sum_{k=1}^{\infty} \frac{[\phi(k, \lambda)]^{m}-(-1)^{m-n} \alpha[\phi(k, \lambda)]^{n}}{1-\alpha}\left|b_{k}\right||z|^{k-1}\right\} \\
& >2(1-\alpha)\left\{1-\sum_{k=j+1}^{\infty} \frac{[\phi(k, \lambda)]^{m}-\alpha[\phi(k, \lambda)]^{n}}{1-\alpha}\left|a_{k}\right|\right. \\
& \left.-\sum_{k=1}^{\infty} \frac{[\phi(k, \lambda)]^{m}-(-1)^{m-n} \alpha[\phi(k, \lambda)]^{n}}{1-\alpha}\left|b_{k}\right|\right\} \\
& \geq 0, \quad \text { Using (6). }
\end{aligned}
$$

The coefficient bound (6) is sharp for the function

$$
\begin{aligned}
f(z)=z & +\sum_{k=j+1}^{\infty} \frac{1-\alpha}{\left([\phi(k, \lambda)]^{m}-\alpha[\phi(k, \lambda)]^{n}\right)} x_{k} z^{k} \\
& +\sum_{k=1}^{\infty} \frac{1-\alpha}{\left([\phi(k, \lambda)]^{m}-(-1)^{m-n} \alpha[\phi(k, \lambda)]^{n}\right)} \overline{y_{k} z^{k}}
\end{aligned}
$$

where $0 \leq \alpha<1,0 \leq \lambda<1, m \in N, n \in N_{0}, m>n$ and

$$
\sum_{k=j+1}^{\infty}\left|x_{k}\right|+\sum_{k=1}^{\infty}\left|y_{k}\right|=1
$$

This completes the proof of Theorem 1 .

In the following theorem, it is proved that the condition (6) is also necessary for the functions $f_{m}=h+\overline{g_{m}}$, where $h$ and $g_{m}$ are of the form (5).

Theorem 2. Let $f_{m}=h+\bar{g}_{m}$ be given by (5). Then $f_{m} \in \bar{S}_{H}^{\lambda}(m, n, j ; \alpha)$ if and only if

$$
\sum_{k=j+1}^{\infty} \frac{[\phi(k, \lambda)]^{m}-\alpha[\phi(k, \lambda)]^{n}}{1-\alpha}\left|a_{k}\right|+\sum_{k=1}^{\infty} \frac{[\phi(k, \lambda)]^{m}-(-1)^{m-n} \alpha[\phi(k, \lambda)]^{n}}{1-\alpha}\left|b_{k}\right| \leq 1
$$

Proof. Since $\bar{S}_{H}^{\lambda}(m, n, j ; \alpha) \subset S_{H}^{\lambda}(m, n, j ; \alpha)$, we only need to prove the "only if" part of the theorem. To this end, for function $f_{m}$ of the form (5), we notice that the condition

$$
\Re\left\{\frac{\Omega^{m} f_{m}(z)}{\Omega^{n} f_{m}(z)}\right\} \geq \alpha
$$


B. Singh, S. Porwal - A new subclass of harmonic univalent functions ...

is equivalent to

$$
\Re\left\{\begin{array}{c}
(1-\alpha) z-\sum_{k=j+1}^{\infty}\left\{(\phi(k, \lambda))^{m}-\alpha(\phi(k, \lambda))^{n}\right\}\left|a_{k}\right| z^{k} \\
+(-1)^{2 m-1} \sum_{k=1}^{\infty}\left\{(\phi(k, \lambda))^{m}-(-1)^{m-n} \alpha(\phi(k, \lambda))^{n}\right\}\left|b_{k}\right| \bar{z}^{k} \\
z-\sum_{k=j+1}^{\infty}(\phi(k, \lambda))^{n}\left|a_{k}\right| z^{k}+(-1)^{m+n-1} \sum_{k=}^{\infty}(\phi(k, \lambda))^{n}\left|b_{k}\right| \bar{z}^{k}
\end{array}\right\} 0 .
$$

The above required condition (10) must hold for all values of $z$ in $U$. Upon choosing the values of $z$ on the positive real axis where $0 \leq z=r<1$, we must have

$$
\begin{gathered}
(1-\alpha)-\sum_{k=j+1}^{\infty}\left\{(\phi(k, \lambda))^{m}-\alpha(\phi(k, \lambda))^{n}\right\}\left|a_{k}\right| r^{k-1} \\
\frac{-\sum_{k=1}^{\infty}\left\{(\phi(k, \lambda))^{m}-(-1)^{m-n} \alpha(\phi(k, \lambda))^{n}\right\}\left|b_{k}\right| r^{k-1}}{1-\sum_{k=j+1}^{\infty}(\phi(k, \lambda))^{n}\left|a_{k}\right| r^{k-1}-(-1)^{m-n} \sum_{k=1}^{\infty}(\phi(k, \lambda))^{n}\left|b_{k}\right| r^{k-1}} \geq 0 .
\end{gathered}
$$

If the condition (9) does not hold then the numerator in (11) is negative for $r$ sufficiently close to 1 . Thus there exist a $z_{0}=r_{0}$ in $(0,1)$ for which the quotient in (11) is negative. This contradicts the required condition for $f_{m} \in \bar{S}_{H}^{\lambda}(m, n, j ; \alpha)$ and so the proof is complete.

We prove the following Theorems 3, 4, 5 and 6 by using techniques adopted by Yalcin [10].

Theorem 3. Let $f_{m}$ be given by (5). Then $f_{m} \in \bar{S}_{H}^{\lambda}(m, n, j ; \alpha)$ if and only if

$$
f_{m}(z)=\sum_{k=1}^{\infty}\left(x_{k} h_{k}(z)+y_{k} g_{m k}(z)\right)
$$

where

$$
\begin{gathered}
h_{1}(z)=z, h_{k}(z)=z-\frac{1-\alpha}{(\phi(k, \lambda))^{m}-\alpha(\phi(k, \lambda))^{m}} z^{k}, \quad(k=j+1, j+2, j+3, \ldots), \\
g_{m k}(z)=z+(-1)^{m-1} \frac{1-\alpha}{(\phi(k, \lambda))^{m}-(-1)^{m-n} \alpha(\phi(k, \lambda))^{n}} \bar{z}^{k}, \quad(k=1,2,3,4, \ldots),
\end{gathered}
$$


B. Singh, S. Porwal - A new subclass of harmonic univalent functions ...

$x_{k} \geq 0, y_{k} \geq 0, \quad \sum_{k=j+1}^{\infty} x_{k}+\sum_{k=1}^{\infty} y_{k}=1-x_{1}$. In particular, the extreme points of $\bar{S}_{H}^{\lambda}(m, n, j ; \alpha)$ are $\left\{h_{k}\right\}$ and $\left\{g_{m k}\right\}$.

Theorem 4. Let $f_{m} \in \bar{S}_{H}^{\lambda}(m, n, j ; \alpha)$. Then for $|z|=r<1$, we have

$$
\begin{aligned}
\left|f_{m}(z)\right| \leq & \left(1+\left|b_{1}\right|\right) r+\left|b_{2}\right| r^{2}+\ldots+\left|b_{j}\right| r^{j}+\frac{1-\alpha}{[\phi(j+1, \lambda)]^{m}-\alpha[\phi(j+1, \lambda)]^{n}} \\
& {\left[1-\frac{1-(-1)^{m-n} \alpha}{1-\alpha}\left|b_{1}\right|-\frac{[\phi(2, \lambda)]^{m}-(-1)^{m-n} \alpha[\phi(2, \lambda)]^{n}}{1-\alpha}\left|b_{2}\right|-\right.} \\
& \left.\ldots-\frac{[\phi(j, \lambda)]^{m}-(-1)^{m-n} \alpha[\phi(j, \lambda)]^{n}}{1-\alpha}\left|b_{j}\right|\right] r^{j+1}, \quad|z|=r<1
\end{aligned}
$$

and

$$
\begin{aligned}
\left|f_{m}(z)\right| \leq & \left(1-\left|b_{1}\right|\right) r-\left|b_{2}\right| r^{2}-\ldots-\left|b_{j}\right| r^{j}-\frac{1-\alpha}{[\phi(j+1, \lambda)]^{m}-\alpha[\phi(j+1, \lambda)]^{n}} \\
& {\left[1-\frac{1-(-1)^{m-n} \alpha}{1-\alpha}\left|b_{1}\right|-\frac{[\phi(2, \lambda)]^{m}-(-1)^{m-n} \alpha[\phi(2, \lambda)]^{n}}{1-\alpha}\left|b_{2}\right|-\right.} \\
& \left.\ldots-\frac{[\phi(j, \lambda)]^{m}-(-1)^{m-n} \alpha[\phi(j, \lambda)]^{n}}{1-\alpha}\left|b_{j}\right|\right] r^{j+1}, \quad|z|=r<1
\end{aligned}
$$

The proof of following theorems are similar to the corresponding results of [3], therefore we only state the results.

Theorem 5. For $0 \leq \beta \leq \alpha<1$ let $f_{m} \in \bar{S}_{H}^{\lambda}(m, n, j ; \alpha)$ and $F_{m} \in \bar{S}_{H}^{\lambda}(m, n, j ; \beta)$. Then $f_{m} * F_{m} \in \bar{S}_{H}^{\lambda}(m, n, j ; \alpha) \subset \bar{S}_{H}^{\lambda}(m, n, j ; \beta)$.

Theorem 6. The class $\bar{S}_{H}^{\lambda}(m, n, j ; \alpha)$ is closed under convex combination.

\section{REFERENCES}

[1] Y. Avci and E. Zlotkiewicz, On harmonic univalent mappings, Ann. Univ. Mariae Curie-Sklodowska Sect. A 44 (1990), 1-7.

[2] J. Clunie and T. Sheil-Small, Harmonic univalent functions, Ann. Acad. Sci. Fen. Series A. I. Math., 9 (1984), 325.

[3] K.K. Dixit and Saurabh Porwal, A new subclass of harmonic univalent functions defined by fractional calculus, General Math., 19(2) (2011), 81-89.

[4] J.M. Jahangiri, Harmonic functions starlike in the unit disk, J. Math. Anal. Appl., 235 (1999), 470- 477.

[5] J.M. Jahangiri, G. Murugusundaramoorthy and K. Vijaya, Salagean-type harmonic univalent functions, South. J. Pure Appl. Math., 2 (2002), 77-82. 
B. Singh, S. Porwal - A new subclass of harmonic univalent functions ...

[6] Saurabh Porwal, A new subclass of harmonic univalent functions associated with fractional calculus operator, Fract. Diff. Calc., 5(1) (2015), 15-24.

[7] Saurabh Porwal and M.K. Aouf, On a new subclass of harmonic univalent functions defined by fractional calculus operator, J. Frac. Calc. Appl., 4 (2013), No. $10,1-12$.

[8] H. Silverman, Harmonic univalent functions with negative coefficients, J. Math. Anal. Appl., 220 (1998), 283-289.

[9] H. Silverman and E.M. Silvia, Subclasses of harmonic functions, New Zealand J. Math., 28 (1999), 275-284.

[10] S. Yalcin, A new class of Salagean-type harmonic univalent functions, Appl. Math. Lett., 18 (2005), 191-198.

Dr. Balvir Singh

Assoc. Professor, Department of Mathematics

R P Degree College, Kamalganj, Farrukhabad-209601

(U.P.) India

email: balvirsingh.rp@gmail.com

Saurabh Porwal

Lecturer Mathematics

Sri Radhey Lal Arya Inter College, Ahen, Hathras

(U.P.) India

email:saurabhjcb@rediffmail.com 